\title{
First Evaluation of Zygomaticus Major Muscle Elastic Properties Using a US Elastography Technique
}

\author{
Redouane Ternifi ${ }^{1}$, Philippe Pouletaut ${ }^{1}$, Stéphanie Dakpé ${ }^{2,3,4,5}$, Sylvie Testelinn ${ }^{2,3,4,5}$, \\ Bernard Devauchelle ${ }^{2,3,4,5}$, Fabrice Charleux ${ }^{6}$, Jean-Marc Constans ${ }^{3,5,7}$, Sabine F. Bensamoun ${ }^{1}$ (D) \\ ${ }^{1}$ Centre de Recherches de Royallieu, UMR CNRS 7338 Biomechanics and Bioengineering, Université de Technologie \\ de Compiègne, Sorbonne University, Compiègne, France; ${ }^{2}$ Maxillofacial Surgery Department, CHU Amiens, France; \\ ${ }^{3}$ EA 7516 Chimère, Université Picardie Jules Verne, Amiens, France; ${ }^{4}$ Fédération Hospitalo-Universitaire SURFACE, \\ Amiens, France; ${ }^{5}$ Institut Faire Faces, Amiens, France; ${ }^{6}$ ACRIM-Polyclinique Saint Côme, Radiologie Médicale, \\ Compiègne, France; ${ }^{7}$ Imagerie et Radiologie Médicale, Amiens, France
}

Correspondence to: Sabine F. Bensamoun, sabine.bensamoun@utc.fr Keywords: Facial Muscle, Shear Wave Elastography, Zygomaticus Major, Texture Analysis, Ultrasound Received: October 15, $2019 \quad$ Accepted: November 17, $2019 \quad$ Published: November 20, 2019

Copyright $\odot 2019$ by author(s) and Scientific Research Publishing Inc.

This work is licensed under the Creative Commons Attribution International License (CC BY 4.0).

http://creativecommons.org/licenses/by/4.0/

\section{Open Access}

\section{ABSTRACT}

Facial expressions are linked to movements of muscles, which can be altered by pathological diseases. Assessment of facial muscle deficits is subjective (palpation) and operator-dependent, and these deficits are currently estimated with clinical scales. Thus, the quantification of facial muscle elastic properties is a key for the clinical adaption and evaluation of treatments for facial paralysis. We herein present a novel application of shear wave elastography (SWE) based on an ultrasound protocol to assess the morphological (thickness and texture) and elastic (Young's modulus) properties of the zygomaticus major (ZM) muscle. Fifteen healthy volunteers underwent SWE tests, and the ultrasound acquisitions were obtained using a new linear transducer (SLH20-6, spatial resolution: $38 \mu \mathrm{m}$ ) and compared to those obtained using an SL10-2 probe (spatial resolution: $50 \mu \mathrm{m}$ ). The probe position was placed along the muscle fiber orientation. A semi-automatic method was developed to quantify the ZM muscle elasticity, and the repeatability was analyzed at one-week intervals. The mean elasticity for the two probes was about $15 \mathrm{kPa}$. The SLH20-6 probe yielded a higher mean elasticity (approximately $6 \mathrm{kPa}$ ) and less homogeneous echogenicity than the SL10-2 probe. Two distinct groups of texture profiles as a function of the transducer were obtained. This study will provide some guidance for clinical practices and will allow the construction of a reference database that could be used to evaluate treatments and develop numerical models of facial expression. 


\section{INTRODUCTION}

Facial expressions are linked to movements of mimic muscles, and the zygomaticus major (ZM) muscle is among the principal muscles involved in producing facial expressions. The $\mathrm{ZM}$ can be altered by pathology (facial paralysis, trauma), which could have serious consequences on the social life of the patient [1]. Facial mimic muscle deficits are assessed by clinical observations and/or palpation and evaluated based on the muscle clinical scales Sunnybrook and the House-Brackmann Score [2], which are used to grade facial paralysis. However, this qualitative approach is insufficient, and these assessments are limited and operator-dependent. Thus, the quantification of facial muscle elastic properties is a key for the clinical adaptation and evaluation of treatments.

Electromyography (EMG) is routinely used in clinical practice to evaluate the physiological activity of the facial nerve in case of paralysis. However, this test may be invasive, if needles are used, and cannot provide the muscle activity of several muscles at once unless several surface electrodes are placed on the face which could be uncomfortable for the patients [3,4]. Recently, surface EMG has been used with inverse model to predict muscle face treatment related function loss [5].

Subsequently, facial motion has been characterized with photography [6] or videoscopy [7]. These methods are difficult to use in clinical routine due to the time required to achieve them for each patient. Movements of the facial mimic muscles have been investigated with motion capture technique and it has been demonstrated that the face markers are able to identify and to quantify the movements [8]. This protocol is still at the research level and could be useful for the rehabilitation of patient suffering of facial paralysis.

Imaging technique such as ultrasonography provides morphological information of different facial muscles such as: length, thickness, area, volume, and echo intensity. Volk et al. [9] have shown higher echo intensity for affected muscle, and structural changes such as atrophy. All of these parameters could be used to better characterize the status of the patient during the disease. Other studies have analyzed the height of the gingival tooth during smile in function of the thickness of the zygomaticus muscle [10].

Imaging techniques, such as ultrasound (US) strain elastography (also called sonoelastography), have revealed facial mimic muscle hardness through displacement measurements under conditions of compression stress [11]. The disadvantage of this technique is that the mechanical properties are assessed based on a hardness scale in color [12]. Since 2008, shear wave elastography (SWE) has been shown to display real-time elastographic output as the shear wave velocity or estimated tissue elasticity $[13,14]$. This technique has been integrated into commercially available, multipurpose, clinical US systems (Aixplorer ${ }^{\mathbb{R}}$ machine) and can be conveniently used in various clinical settings. SWE produces elastographic color maps capable of providing quantitative elasticity measurements in a user-defined region of interest (ROI) at different sites, such as superficial or deep soft tissues. Importantly, in contrast to strain elastography, SWE does not require any operator compression or a mechanical source for the stress generation and, hence, is a more reproducible technique [15]. SWE has been applied to head and neck tissues and, more specifically, to evaluate the elastic properties of the masseter muscle $[16,17]$, cervical muscles $[18,19]$, salivary glands [20] and thyroid [21, 22]. However, in vivo data on the elastic properties of healthy and pathologically affected mimic muscles are lacking.

Currently, no techniques providing quantitative values regarding the elastic properties of mimic muscles are described in the literature. These data could elucidate the residual muscle quality for maxillofacial surgeons, thus aiding in the selection of a surgical technique or injection in patients suffering from facial palsy. Furthermore, elastic properties are important because in cases of facial nerve paralysis or cofacial muscle contraction, botulinum toxin injections are performed inside the ZM muscle to correct the symmetry of the patient's smile. Thus, the novelty of the present study was to propose an efficient, reproducible, US elastography imaging protocol that could be incorporated into patient care to quantify the elastic properties of the ZM. 


\section{MATERIALS AND METHODS}

\subsection{Participants}

In this study, fifteen healthy volunteers ( 8 women, 7 men, age range: 20 to 29 , mean age: $24.8 \pm 3.2$ years) with no facial muscle damage and no history of facial muscle disease were recruited. Each subject underwent a shear wave elastography (SWE) test and only volunteers satisfying the following inclusion (male or female aged $\geq 18$ years, person enrolled in a social security scheme, patient able to understand the briefing note and giving his written consent) and exclusion (ZM thickness $>1 \mathrm{~mm}$, bifid (i.e. divided muscle) and fibrotic muscle structure) criteria were selected for a SWE exam. This study was approved by the institutional review board of Sud Est VI (\#2018-A02389-46), and informed consent was obtained from the participants.

\subsection{Shear Wave Elastography (SWE)}

The subjects laid on their sides in a relaxed, reclined position with pillows under their heads. The volunteers were asked to relax their mouths to avoid any contraction of the ZM. All subjects underwent US examination of the left ZM using an Aixplorer Multiwave ${ }^{\mathrm{TM}}$ System (Supersonic Imagine, Aix-en-Provence, France), which was performed by the same operator (with 7 years of experience with US). Two linear transducers were used for each participant to visualize the $\mathrm{ZM}$ at different resolutions. The first probe had a $2.38 \mathrm{~cm}$ footprint (SuperLinear ${ }^{\mathrm{TM}} \mathrm{SLH} 20-6$, with 192 elements and a bandwidth of $6-20 \mathrm{MHz}$ ), and the second linear transducer had a $3.2 \mathrm{~cm}$ footprint (SuperLinear ${ }^{\mathrm{TM}}$ SL10-2, with 192 elements and a bandwidth of $2-10 \mathrm{MHz}$ ). This machine generates pushing pulses and imaging pulses that propagate within tissue. The imaging pulse creates a high-quality B-mode image showing the anatomical structure inside the face. The parameter settings of the B-mode images were as follows: gain, 50\%; pulse repetition frequency, $20 \mathrm{kHz}$; and axial spatial resolution, $38 \mu \mathrm{m}$ for the SLH20-6 probe and $50 \mu \mathrm{m}$ for the SL10-2 probe (Figure 1). The pushing pulses produce shear waves in the tissues which are represented through a quantitative color-coded map of tissue elasticity. A chromatic scale, progressing from blue to red, represents the lowest to the highest elasticity values, respectively. The parameter settings for the shear wave elasticity mapping, for both probes, were as follows: musculoskeletal preset, resolution mode enabled, tissue tuner: $1540 \mathrm{~m} \cdot \mathrm{s}^{-1}$, pulse duration: $600 \mu \mathrm{s}$, depth: $2 \mathrm{~cm}$, gain: $50 \%$, dynamic range: $60 \mathrm{~dB}$, and spatial resolution: $0.9 \mathrm{~mm}$. The range of elasticity (Young's modulus, E) was set between $0 \mathrm{kPa}$ and $180 \mathrm{kPa}$; assuming that the ZM muscle was linearly elastic, isotropic, homogeneous and incompressible, this range corresponds to a shear wave velocity range of $0-7.7 \mathrm{~m} \cdot \mathrm{s}^{-1}$.

To image the same area of the ZM muscle, the zygomatic bone was used as a landmark. From this point, the probe was pointed towards the corner of the mouth, tilted obliquely (approximately 45 degrees) and aligned with respect to muscle fiber orientation. To scan the same belly area of the ZM muscle for all subjects, a visual control was performed on the B-mode images using zygomatic bone as the reference tissue. Nevertheless, the morphology (length and thickness) of the ZM could vary among subjects in the cohort. To ensure that the same ZM region was scanned, a box $(1 \mathrm{~cm} \times 2 \mathrm{~cm})$ was placed on the B-mode image containing the bone and the belly portion of the ZM (Figure 1).

\subsection{Data Processing}

The Aixplorer Multiwave ${ }^{\mathrm{TM}}$ System allows for the automatic quantification of elasticity by placement of a rectangular box. DICOM (Digital Imaging and Communications in Medicine) data were exported and analyzed using custom MATLAB scripts (The MathWorks Inc., Natick, MA, USA). To quantify the SWE elasticity of the ZM, a semi-automatic method was developed using ImageJ 1.46/Java 8 software (National Institute of Health, Bethesda, MD, United States) [23]. A mask was manually traced in the belly region of the $\mathrm{ZM}$ from the $\mathrm{B}$-mode image to delineate the region of interest (ROI) along the $\mathrm{ZM}$, which was then superimposed onto B-mode and elasticity images (Figure 1 ).

From this ROI, the mean $\mathrm{E}$ values were calculated. In addition to the mean elasticity parameters, the 
(a)
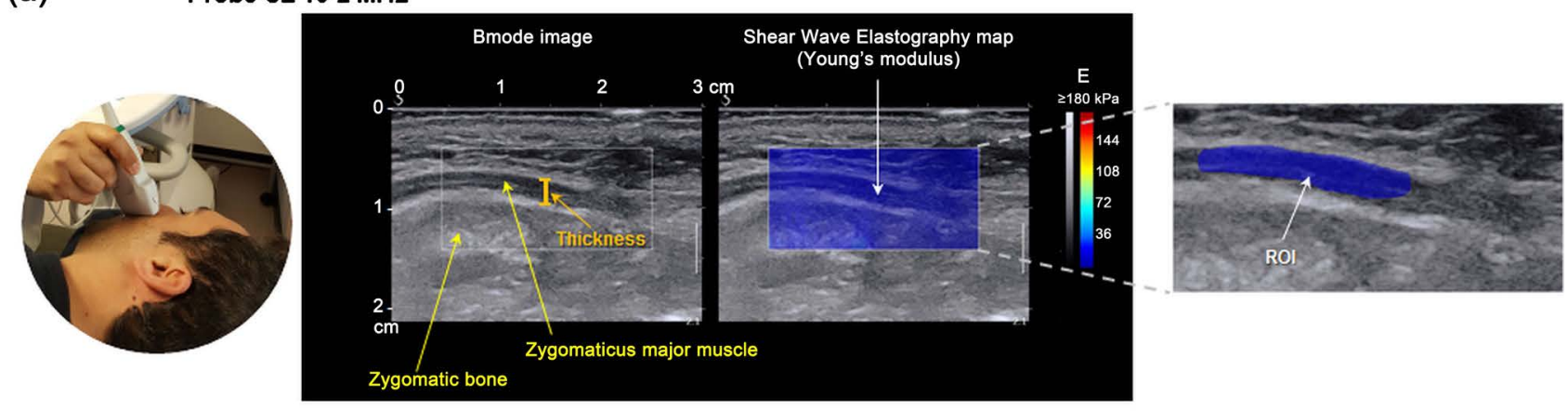

(b) Probe SLH 20-6 MHz
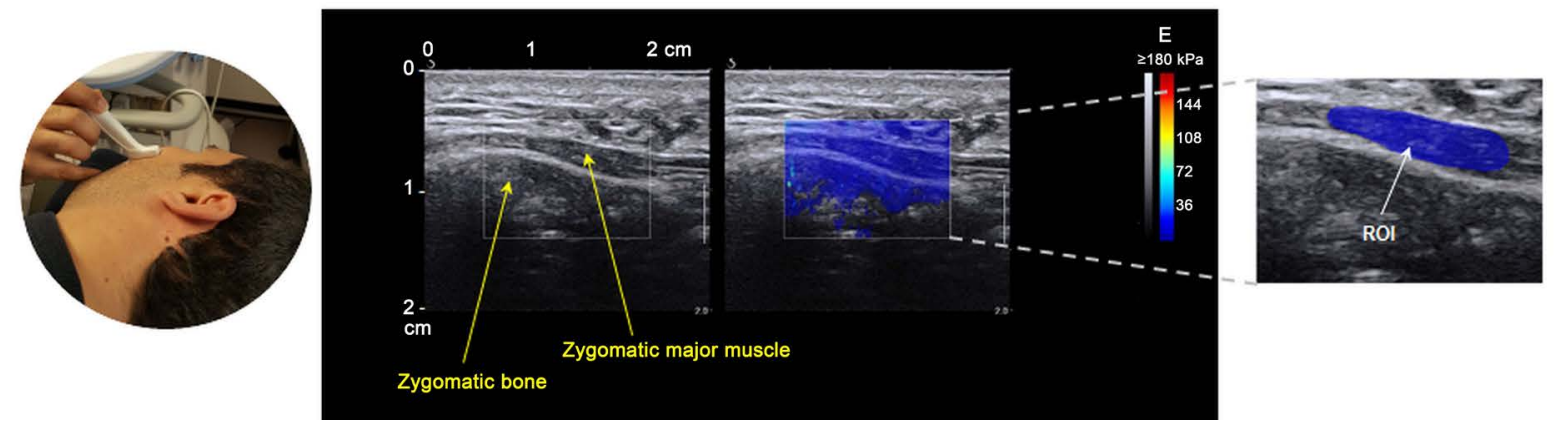

Figure 1. SWE measurement using the SL 10-2 probe (a) and the SuperLinear ${ }^{\mathrm{TM}}$ SLH20-6 probe (b) over the ZM muscle. The color elastogram in the rectangular box shows a homogeneous blue color, indicating the uniformity of the soft tissues in a range of Young's modulus (E) values up to $180 \mathrm{kPa}$. The ROI on the right represents the measurement ROI over the $\mathrm{ZM}$ muscle. The red line on the grayscale image indicates the measurement of the zygomaticus MT.

mean muscle thickness (MT) of the ZM was manually estimated with the tracing tool in ImageJ software. From the ROI on the B-mode image, vertical lines were drawn every 3 millimeters along the $\mathrm{ZM}$. The mean echo intensity (EI) data were also measured in gray-scale (0-255) from the ROI in the B-mode image.

To compare the images produced by the two transducers, histograms in the ROIs were further analyzed. The following parameters were calculated: mean, standard deviation and coefficient of variation (ratio of the standard deviation to the mean). The coefficient of variation was used to assess the variability of the measurements in the ROI.

The ROIs extracted from the B-mode images were also used for the texture analysis using Mazda 4.6 software [24], which includes several texture analysis methods. In this study, gray-level histogram parameters and co-occurrence parameters were used. Selection of the texture parameters based on Fisher's coefficient [25] was performed to identify the three most significant parameters able to discriminate the B-mode images generated by the two transducers. Fisher's coefficient is defined as the ratio of between-class variance to within-class variance.

The repeatability was analyzed by assessing images from five participants who underwent SWE examinations at one-week intervals. The total duration of each experiment is 10 minutes. During these two US sessions, the measurements (thickness and elasticity) were performed twice. The value of the root mean square of the difference (RMSD) of the two successive measurements was calculated as follows:

$$
\operatorname{RMSD}=\sqrt{\frac{1}{N} \sum_{N=1}^{n}\left[\operatorname{Meas}\left(t_{n 1}\right)-\operatorname{Meas}\left(t_{n 2}\right)\right]^{2}}
$$


where Meas represents the measurement value (thickness or elasticity) and $N$ is the number of subjects examined by the operator.

\subsection{Statistical Analysis}

The XLSTAT $^{\mathrm{TM}}$ software package (Addinsoft, Paris, France) was used to perform all statistical analyses. All parameters are expressed as the mean \pm standard deviation. Nonparametric two-sample Kolmogorov-Smirnov tests were performed to compare the elasticity and B-mode (thickness, texture and EI) values between the two transducers. Statistical analyses were considered significant at $\mathrm{p}<0.05$.

\section{RESULTS}

\subsection{SWE Morphological, Textural and Elastic Properties}

Figure 2 and Table 1 summarize the ZM muscle thickness (MT), echo intensity (EI) and Young's modulus (E) results. The MT and EI did not significantly differ between the two probes. However, the mean elasticity achieved with the SLH20-6 probe was significantly higher $(18.3 \pm 3.7 \mathrm{kPa})$ than that achieved with the SL10-2 probe $(12 \pm 4.3 \mathrm{kPa})$. The range (from $4.6 \mathrm{kPa}$ to $20.5 \mathrm{kPa}$ ) of the elastic data obtained with the SL10-2 probe was larger than that obtained with the SLH20-6 probe (from $13.3 \mathrm{kPa}$ to 27.6 $\mathrm{kPa})$.

The coefficient of variation in the B-mode images obtained with the SLH20-6 probe showed a larger range of data than that obtained with the SL10-2 probe, revealing less homogeneous echogenicity

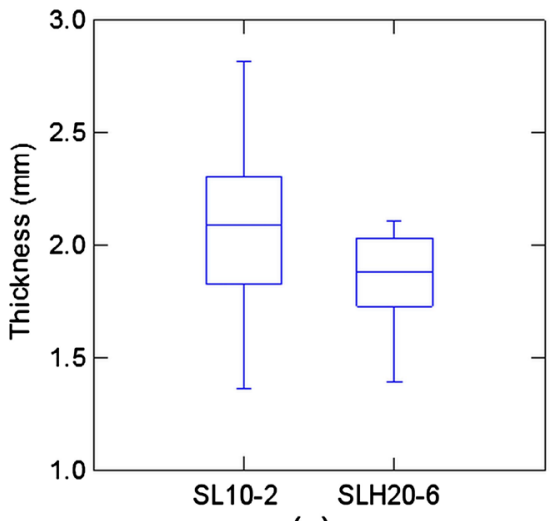

(a)

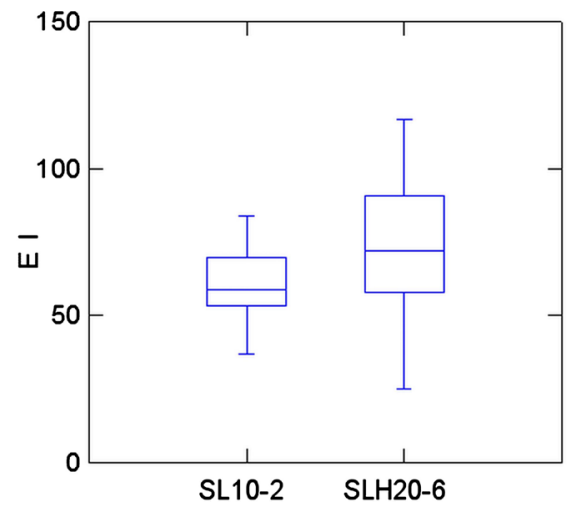

(b)

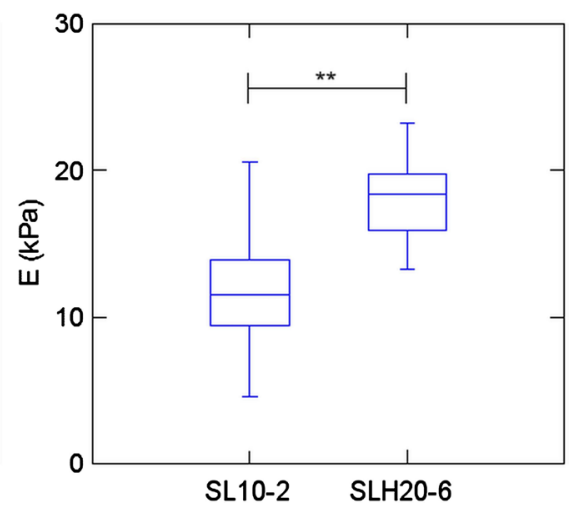

(c)

Figure 2. Zygomaticus muscle thickness (MT), echo intensity (EI) and Young's modulus (E) data for 15 healthy volunteers obtained using two linear transducers, SL10-2 and SLH20-6 (** $\mathrm{p}<0.001)$.

Table 1. Comparison of the means of the zygomaticus Muscle Thickness (MT), Echo Intensity (EI), and Young modulus (E) data in 15 healthy volunteers using two transducers.

\begin{tabular}{|c|c|c|c|c|c|c|c|c|c|c|c|}
\hline \multirow{3}{*}{ Parameter } & \multicolumn{10}{|c|}{ Transducer } & \multirow{3}{*}{ P-value ${ }^{*}$} \\
\hline & \multicolumn{5}{|c|}{ SL10-2 } & \multicolumn{5}{|c|}{ SLH20-6 } & \\
\hline & Median & Mean & SD & Min & Max & Median & Mean & SD & Min & $\operatorname{Max}$ & \\
\hline $\mathrm{MT}(\mathrm{mm})$ & 2.09 & 2.07 & 0.41 & 1.36 & 2.82 & 1.88 & 1.89 & 0.36 & 1.39 & 2.89 & 0.16 \\
\hline EI & 58.9 & 66.6 & 25.2 & 37.1 & 131.2 & 72.1 & 73.1 & 27.2 & 24.9 & 117.0 & 0.16 \\
\hline $\mathrm{E}(\mathrm{kPa})$ & 11.5 & 12.0 & 4.3 & 4.6 & 20.5 & 18.4 & 18.3 & 3.7 & 13.3 & 27.6 & 0.001 \\
\hline
\end{tabular}

Significant differences at the $5 \%$ level are shown in bold. 
(Figure 3(a)). Conversely, the coefficient of variation in elasticity obtained with the SLH20-6 probe showed a smaller range of data than that obtained with the SL10-2 probe, demonstrating a more stable result (Figure 3(b)).

Analysis of the textures of the B-mode images provided the following three discriminant texture parameters as determined by Fisher's coefficient (Figure 4): parameters of the autoregressive model (sigma and theta1) and the co-occurrence value of correlation $S(1,0)$. Figure 4 shows the results of comparing the texture profiles of the SL10-2 probe (labeled \#1) and the SLH20-6 probe (labeled \#2).

Two distinct groups of texture profiles as a function of the transducer were obtained. These results demonstrate that the probes were able to differentiate the ZM texture profiles.

\subsection{Repeatability of the US Study}

The root mean square of the difference (RMSD) of the two successive measurements made by the operator regarding the zygomaticus MT measurements was $0.10 \mathrm{~mm}$ with the SL10-2 probe and $0.07 \mathrm{~mm}$

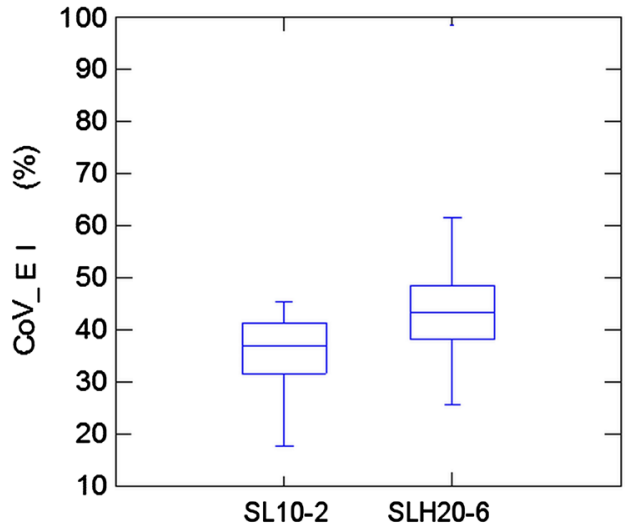

(a)

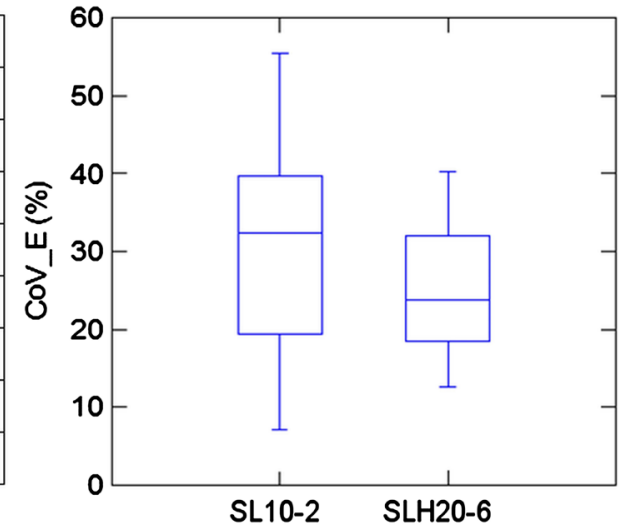

(b)

Figure 3. Coefficients of variation (CoV) (\%) of the echo intensity (EI) and Young's modulus (E) of the ZM muscles in 15 healthy volunteers obtained using two transducers (SL10-2 and SLH20-6).

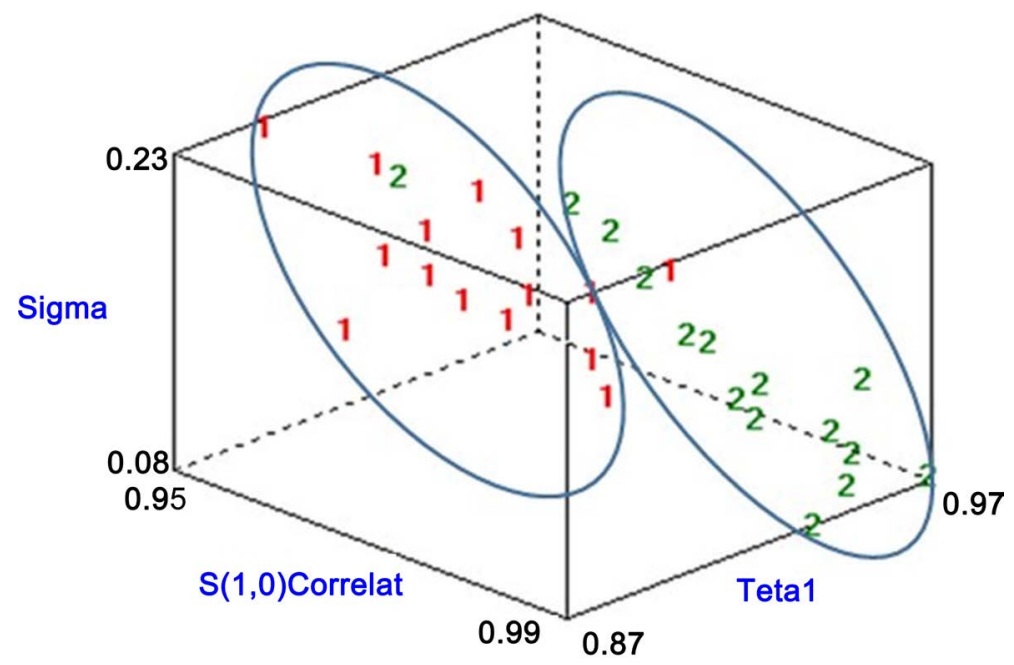

Figure 4. Raw data of the following three most discriminant texture parameters obtained by Fisher's coefficient: parameters of the autoregressive model (sigma and theta1) and the co-occurrence value of correlation S $(1,0)$. Measurements performed with the SL10-2 transducer are labeled "1", and those performed with the SLH20-6 transducer are labeled "2". 
with the SLH20-6 probe. The intraoperator repeatability of the MT measurements made by the two probes were similar.

The RMSD of the intraoperative repeatability of the $\mathrm{E}$ measurements of the $\mathrm{ZM}$ muscle was $0.25 \mathrm{kPa}$ with the SL10-2 probe and $1.35 \mathrm{kPa}$ with the SLH20-6 probe. The measurement of E with the SL10-2 probe appeared to be more repeatable than that with the SLH20-6 probe. The mean absolute value of the difference in two successive measurements of $\mathrm{E}$ between the two probes remained less than $10 \%$ (2.2\% with the SL10-2 probe and 8.5\% with the SLH20-6 probe).

\section{DISCUSSION}

Quantification of the elastic properties of superficial facial muscles with conventional clinical tools is challenging, and the demand for an appropriate technique in the facial field is increasing. An important advantage of the US technique is its ability to dynamically analyze, at different levels of stretch, the ZM muscle, which can be used during rehabilitation. In addition, using an imaging tool, such as US, for guidance during injections could allow for objective monitoring of the effects of the toxin on the structural and elastic properties of the muscle. The originality of the study was to provide referent elastic values of the healthy ZM muscle and to raise the awareness of radiologists using US elastography technique.

The new SLH20-6 probe, commercialized for hand musculoskeletal tissue [26], offers a higher resolution than the SL10-2 probe and we demonstrated that this probe could also be used for the tiny mimic muscles. Moreover, this SLH20-6 probe is capable of revealing changes at the microstructural level through the measurements of textural parameters which could become future biomarkers of the mimic muscles. The SLH20-6 probe represents the best use to characterize the morphological properties of tiny and superficial muscle.

We cautiously avoided manual compression by controlling the thickness of the ZM on the B-mode images. This experimental condition was validated by the thickness results, which were in the same range as the thickness of healthy ZM muscles as measured by MRI $[27,28]$.

A semi-automatic method was developed to quantify the elastic properties using both US probes in the same range as those reported in the literature for other facial muscles (masseter) [29] and head and neck muscles $[17,19]$ using the same SWE method. These results show the feasibility of using US elastography for diagnostic purposes or for evaluating therapeutic interventions performed on the ZM muscle. The present study has demonstrated that the selected probe will influence the ZM elasticity measurement. This variation of elasticity could be due to the frequency and to the characteristics of the probe as well as the tissue properties.

In the literature Franchi-Abella et al. [30] have demonstrated that, by using different ultrasound frequencies on a phantom, mimicking the biological tissue, different elasticity values have been obtained. This similar result showed that the elastic properties are dependent of the frequency. Concerning the characteristics of the probe, it is well known that the generation of shear waves depends on the type of the pushing pulse emitted by the probe (characteristic of the piezos) so that the shear wave has a different bandwidth from one probe to another. In our study, according to the specifications of the probes, different ranges of bandwidth were intrinsically used (from 70 to $800 \mathrm{~Hz}$ for the SLH20-6 probe and from 70 to 680 $\mathrm{Hz}$ for the SL10-2 probe) inducing possible discrepancy in elasticity measurement. Shear wave spectral content is also related to the material properties of the medium, which could induce a dispersion phenomenon due to the presence of the muscle tissue that filters more or less specific frequencies. The elasticity in many biological tissues has been shown to be viscoelastic and to follow a power law with frequency and can be represented by a springpot model [31]. Thus, a probe emitting waves with a higher frequency tends to yield a higher value of elasticity. In addition, anisotropy of the muscle and the "small" nature of the ZM structure relative to the associated shear wave length could contribute to the dispersion of the elasticities between probes. This information could raise the awareness of radiologists or facial surgeons on diagnostic results.

Another crucial element for developing the mimic muscle SWE protocol was to identify the probe 
position with respect to muscle fiber orientation. Muscle behavior is anisotropic, as the highest elasticity values are yielded when the probe is aligned with the muscle fiber, and the lowest values are obtained when the probe is oriented across the muscle fiber [32].

\section{CONCLUSION}

The present study is the first to demonstrate the utility of US elastography in the regular care of facial palsy patients and paves the way for other applications. Development of an US protocol will allow the construction of a reference database composed of the morphological and elastic properties of mimic muscles. These facial quantitative data will enable the adaptation and evaluation of treatments. Future research could be conducted on ZM with different morphologies (bifid, fibrous, etc.), thicknesses and in different state (stretch and contracted). In addition to clinical applications, these values could be used for the development of numerical facial expression models $[28,33]$ using the true in vivo characteristics of the zygomaticus muscle.

\section{ACKNOWLEDGEMENTS}

This work was supported by Hauts-de-France Region and CPER Santé.

\section{CONFLICTS OF INTEREST}

The authors declare no conflicts of interest regarding the publication of this paper.

\section{REFERENCES}

1. Devauchelle, B., Badet, L., Lengelé, B., Morelon, E., Testelin, S., Michallet, M., D’Hauthuille, C. and Dubernard, J.-M. (2006) First Human Face Allograft: Early Report. The Lancet, 368, 203-209. https://doi.org/10.1016/S0140-6736(06)68935-6

2. Kanerva, M., Poussa, T. and Pitkäranta, A. (2006) Sunnybrook and House-Brackmann Facial Grading Systems: Intrarater Repeatability and Interrater Agreement. Otolaryngology-Head and Neck Surgery, 135, 865-871. https://doi.org/10.1016/j.otohns.2006.05.748

3. Schumann, N.P., Bongers, K., Guntinas-Lichius, O. and Scholle, H.C. (2010) Facial Muscle Activation Patterns in Healthy Male Humans: A Multi-Channel Surface EMG Study. Journal of Neuroscience Methods, 187, 120-128. https://doi.org/10.1016/j.jneumeth.2009.12.019

4. Lapatki, B.G., Stegeman, D.F. and Jonas, I.E. (2003) A Surface EMG Electrode for the Simultaneous Observation of Multiple Facial Muscles. Journal of Neuroscience Methods, 123, 117-128. https://doi.org/10.1016/S0165-0270(02)00323-0

5. Eskes, M., Balm, A.J., Van Alphen, M.J., Smeele, L.E., Stavness, I. and Van Der Heijden, F. (2017) sEMG-Assisted Inverse Modelling of 3D Lip Movement: A Feasibility Study towards Person-Specific Modelling. Scientific Reports, 7, 17729. https://doi.org/10.1038/s41598-017-17790-4

6. Neely, J. G., Cheung, J.Y., Wood, M., Byers, J. and Rogerson, A. (1992) Computerized Quantitative Dynamic Analysis of Facial Motion in the Paralyzed and Synkinetic Face. The American Journal of Otology, 13, 97-107.

7. Linstrom, C.J., Silverman, C.A. and Susman, W.M. (2000) Facial-Motion Analysis with a Video and Computer System: A Preliminary Report. Otology \& Neurotology, 21, 123-129.

https://doi.org/10.1016/S0196-0709(00)80086-1

8. Ben Mansour, K., Sarhan, F.R., Neiva, C., Godard, C., Devauchelle, B., Marin, F. and Dakpé, S. (2014) Analysis of Mimic Facial Movements Based on Motion Capture. Computer Methods in Biomechanics and Biomedical Engineering, 17, 78-79. https://doi.org/10.1080/10255842.2014.931142

9. Volk, G.F., Pohlmann, M., Sauer, M., Finkensieper, M. and Guntinas-Lichius, O. (2014) Quantitative Ultraso- 
nography of Facial Muscles in Patients with Chronic Facial Palsy. Muscle \& Nerve, 50, 358-365. https://doi.org/10.1002/mus.24154

10. McAlister, R.W., Harkness, E.M. and Nicoll, J.J. (1998) An Ultrasound Investigation of the Lip Levator Musculature. The European Journal of Orthodontics, 20, 713-720. https://doi.org/10.1093/ejo/20.6.713

11. Ariji, Y., Nakayama, M., Nishiyama, W., Nozawa, M. and Ariji, E. (2015) Shear-Wave Sonoelastography for Assessing Masseter Muscle Hardness in Comparison with Strain Sonoelastography: Study with Phantoms and Healthy Volunteers. Dentomaxillofacial Radiology, 45, 20150251. https://doi.org/10.1259/dmfr.20150251

12. Ariji, Y., Nakayama, M., Nishiyama, W. and Ariji, E. (2014) Applications of Sonographic Elastography to the Oral and Maxillofacial Region. Journal of Radiology \& Radiation Therapy, 2, 1049.

13. Bercoff, J., Tanter, M. and Fink, M. (2004) Supersonic Shear Imaging: A New Technique for Soft Tissue Elasticity Mapping. IEEE Transactions on Ultrasonics, Ferroelectrics and Frequency Control, 51, 396-409. https://doi.org/10.1109/TUFFC.2004.1295425

14. Tanter, M., Bercoff, J., Athanasiou, A., Deffieux, T., Gennisson, J.-L., Montaldo, G., Muller, M., Tardivon, A. and Fink, M. (2008) Quantitative Assessment of Breast Lesion Viscoelasticity: Initial Clinical Results Using Supersonic Shear Imaging. Ultrasound in Medicine \& Biology, 34, 1373-1386. https://doi.org/10.1016/j.ultrasmedbio.2008.02.002

15. Kim, H.J., Kim, S.M., Kim, B., Yun, B.L., Jang, M., Ko, Y., Lee, S.H., Jeong, H., Chang, J.M. and Cho, N. (2018) Comparison of Strain and Shear Wave Elastography for Qualitative and Quantitative Assessment of Breast Masses in the Same Population. Scientific Reports, 8, 1-11. https://doi.org/10.1038/s41598-018-24377-0

16. Ewertsen, C., Carlsen, J., Perveez, M.A. and Schytz, H. (2018) Reference Values for Shear Wave Elastography of Neck and Shoulder Muscles in Healthy Individuals. Ultrasound International Open, 4, E23-E29. https://doi.org/10.1055/s-0044-102013

17. Herman, J., Sedlackova, Z., Vachutka, J., Furst, T., Salzman, R. and Vomacka, J. (2017) Shear Wave Elastography Parameters of Normal Soft Tissues of the Neck. Biomedical Papers, 161, 320-325. https://doi.org/10.5507/bp.2017.024

18. Dieterich, A.V. andrade, R.J., Le Sant, G., Falla, D., Petzke, F., Hug, F. and Nordez, A. (2017) Shear Wave Elastography Reveals Different Degrees of Passive and Active Stiffness of the Neck Extensor Muscles. European Journal of Applied Physiology, 117, 171-178. https://doi.org/10.1007/s00421-016-3509-5

19. Liu, K.H., Bhatia, K., Chu, W., He, L.T., Leung, S.F. and Ahuja, A.T. (2015) Shear Wave Elastography-A New Quantitative Assessment of Post-Irradiation Neck Fibrosis. Ultraschall in der Medizin, 36, 348-354. https://doi.org/10.1055/s-0034-1366364

20. Mantsopoulos, K., Klintworth, N., Iro, H. and Bozzato, A. (2015) Applicability of Shear Wave Elastography of the Major Salivary Glands: Values in Healthy Patients and Effects of Gender, Smoking and Pre-Compression. Ultrasound in Medicine \& Biology, 41, 2310-2318. https://doi.org/10.1016/j.ultrasmedbio.2015.04.015

21. Bhatia, K., Tong, C.S.L., Cho, C.C.M., Yuen, E.H.Y., Lee, J. and Ahuja, A.T. (2012) Reliability of Shear Wave Ultrasound Elastography for Neck Lesions Identified in Routine Clinical Practice. Ultraschall in der Medizin, 33, 463-468. https://doi.org/10.1055/s-0032-1325428

22. Habibi, H.A., Durmaz, E.S.M., Qarayeva, V., Kandemirli, S.G., Kalyoncu Ucar, A., Aslan, M., Apaydin, G., Kurugoglu, S. and Adaletli, I. (2018) Quantitative Assessment of Thyroid, Submandibular and Parotid Glands Elasticity with Shear-Wave Elastography in Children. Ultrasound Quarterly, 34, 58-61. https://doi.org/10.1097/RUQ.0000000000000352

23. Schneider, C.A., Rasband, W.S. and Eliceiri, K.W. (2012) NIH Image to ImageJ: 25 Years of Image Analysis. Nat Methods, 9, 671-675. https://doi.org/10.1038/nmeth.2089 
24. Szczypiński, P.M., Strzelecki, M., Materka, A. and Klepaczko, A. (2009) MaZda-A Software Package for Image Texture Analysis. Computer Methods and Programs in Biomedicine, 94, 66-76. https://doi.org/10.1016/j.cmpb.2008.08.005

25. Schürmann, J. (1996) Pattern Classification: A Unified View of Statistical and Neural Approaches. Wiley, New York, 1-17.

26. SuperTransducersTM Brochure, Supersonic Imagine (2015) Aixplorer.

27. Dao, T.T., Dakpé, S., Pouletaut, P., Devauchelle, B. and Ho Ba Tho, M.C. (2013) Facial Mimics Simulation Using MRI and Finite Element Analysis. Annual International Conference of the IEEE Engineering in Medicine and Biology Society, 2013, 4585-4588.

28. Fan, A.-X., Dakpé, S., Dao, T.T., Pouletaut, P., Rachik, M. and Tho, M.C.H.B. (2017) MRI-Based Finite Element Modeling of Facial Mimics: A Case Study on the Paired Zygomaticus Major Muscles. Computer Methods in Biomechanics and Biomedical Engineering, 20, 919-928. https://doi.org/10.1080/10255842.2017.1305363

29. Arda, K., Ciledag, N., Aktas, E., Arıbas, B.K. and Köse, K. (2011) Quantitative Assessment of Normal Soft-Tissue Elasticity Using Shear-Wave Ultrasound Elastography. American Journal of Roentgenology, 197, 532-536. https://doi.org/10.2214/AJR.10.5449

30. Franchi-Abella, S., Elie, C. and Correas, J.-M. (2017) Performances and Limitations of Several Ultrasound-Based Elastography Techniques: A Phantom Study. Ultrasound in Medicine \& Biology, 43, 2402-2415. https://doi.org/10.1016/j.ultrasmedbio.2017.06.008

31. Palmer, B.M., Tanner, B.C.W., Toth, M.J. and Miller, M.S. (2013) An Inverse Power-Law Distribution of Molecular Bond Lifetimes Predicts Fractional Derivative Viscoelasticity in Biological Tissue. Biophysical Journal, 104, 2540-2552. https://doi.org/10.1016/j.bpj.2013.04.045

32. Gennisson, J.-L., Deffieux, T., Macé, E., Montaldo, G., Fink, M. and Tanter, M. (2010) Viscoelastic and Anisotropic Mechanical Properties of in Vivo Muscle Tissue Assessed by Supersonic Shear Imaging. Ultrasound in Medicine \& Biology, 36, 789-801. https://doi.org/10.1016/j.ultrasmedbio.2010.02.013

33. Esteve-Altava, B., Diogo, R., Smith, C., Boughner, J.C. and Rasskin-Gutman, D. (2015) Anatomical Networks Reveal the Musculoskeletal Modularity of the Human Head. Scientific Reports, 5, 8298. https://doi.org/10.1038/srep08298 